\title{
TRANS-CULTURAL, CROSS-GENERIC AND COMPARATIVE ANALYSES
}

